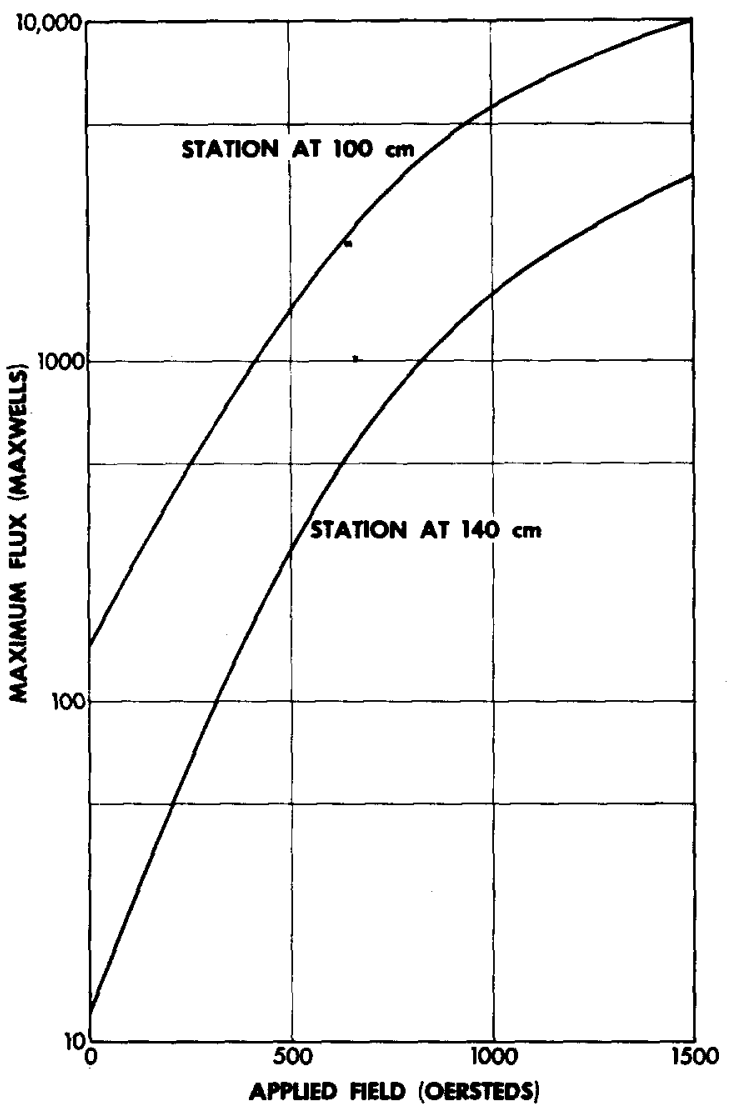

Fig. 4. Effect of applied magnetic field on loop signals at two positions along shock tube.

tively, from the arc chamber. The external field was obtained using a dc power supply and remained constant during the discharge.

The present results, together with those of Scott and Wenzel, ${ }^{1}$ indicate that a traveling magnetic field is produced when a moving plasma interacts with the radial component of an externally imposed magnetic field. In both cases the sign of the signal was reversed when the field on the arc chamber was reversed. The observations suggest that the transport and diffusion effects be examined by considering the induction equation

$$
\partial H / \partial t=\nabla \times(v \times H)+\eta \nabla^{2} H .
$$

In a moving coordinate system it reduces to the diffusion equation

$$
\partial H / \partial t=\eta \nabla^{2} H,
$$

where

$$
\eta=(4 \pi \mu \sigma)^{-1},
$$

from which the decay time is $\tau=4 \pi \mu \sigma L^{2}$. This agrees with the experimentally observed result that the decay time is independent of the externally applied field. From the experimental values $\tau=5.5 \mu \mathrm{sec}$ and $L=15 \mathrm{~cm}$, one obtains $\eta \approx 4 \times 10^{7} \mathrm{~cm}^{2} / \mathrm{sec}$. This value seems reasonable for the observed shock velocity. As a comparison, in the solar photosphere $\eta \approx 10^{8} \mathrm{~cm}^{2} / \mathrm{sec}$.
Consider the transport effect as compared with the diffusion effect by defining the magnetic Reynolds number in the usual way.

$$
R_{m}=\frac{v H / L}{\eta\left(H / L^{2}\right)}=\frac{v L}{\eta} .
$$

For the present case, $R_{m} \approx 2$ which agrees with the experimental observation that the moving field is nearly "frozen" in the gas.

1 F. R. Scott and R. F. Wenzel, Phys. Fluids 2, 609 (1959).

${ }^{2}$ V. Josephson, J. Appl. Phys. 29, 30 (1958).

\section{Electrical Conductivity of a Partially Ionized Gas}

Gordon L, CANN

Guggenheim Aeronautical Laboratory, California Institute of Technology, Pasadena, California (Received July 25, 1960)

W HILE investigating the energy transfer processes occurring between an electric arc and the surrounding gas, ${ }^{1}$ it was found desirable to study in detail the transport properties of species diffusion and thermal flux in a partially ionized monatomic gas in the presence of a magnetic field.

By using the disciplines of irreversible thermodynamics and kinetic theory, ${ }^{2,8}$ the following equations are derived for the electron and ion motion relative to the mass velocity of the gas:

$\mathbf{u}_{\varepsilon}+\gamma(\beta+\epsilon-\eta) \mathbf{u}_{\varepsilon} \times \mathbf{B}$

$$
\begin{aligned}
& -\gamma^{2}[(\beta+\epsilon) \eta-\epsilon]\left(\mathbf{u}_{e} \times \mathbf{B}\right) \times \mathrm{B} \\
= & -\gamma(\beta+\epsilon-1) \mathbf{F}_{1}-\gamma \mathbf{F}_{2} \\
& +\gamma^{2}[(\beta+\epsilon) \eta-\epsilon] \mathbf{F}_{1} \times \mathbf{B}
\end{aligned}
$$

$\mathbf{u}_{I}+\gamma(\boldsymbol{\beta}+\epsilon-\eta) \mathbf{u}_{I} \times \mathbf{B}$

$$
\begin{aligned}
& -\gamma^{2}[(\beta+\epsilon) \eta-\epsilon]\left(\mathbf{u}_{1} \times \mathbf{B}\right) \times \mathbf{B} \\
= & \gamma\left(\beta_{1}+1-\epsilon\right) \mathbf{F}_{1}-\gamma \eta \mathbf{F}_{2} \\
& +\gamma^{2}[(\beta+\epsilon) \eta-\epsilon]\left(\mathbf{F}_{1}-\mathbf{F}_{2}\right) \times \mathbf{B}
\end{aligned}
$$

with

$$
\begin{gathered}
\mathbf{F}_{1}=\mathbf{E}+\frac{k T}{|e|} \nabla \ln p_{e}+\mathbf{u}_{0} \times \mathbf{B}+\frac{k T}{|e|} k_{e} \nabla \ln T \\
\mathbf{F}_{2}=\frac{k T}{|e|} \nabla \ln \frac{p_{e} p_{I}}{p_{a}}+\frac{k T}{|e|}\left(k_{e}-k_{I}\right) \nabla \ln T \\
\gamma \beta=\frac{\sigma_{s p}}{|e| n_{I} \eta},
\end{gathered}
$$




$$
\begin{aligned}
& \beta=\frac{\left(1+n_{a} / n_{T}\right)^{2}}{n_{a} / n_{T}} \frac{q_{I a} f_{e 1}}{q_{e I} f_{J a}}\left(\frac{2 m_{a}}{m_{e}}\right)^{\frac{1}{2}}, \\
& \epsilon=n_{e} / n_{I}, \\
& \eta=1+\beta_{I}=1+\frac{n_{a}}{n_{I}} \frac{q_{e a}}{q_{I e}} \frac{f_{I e}}{f_{e a}},
\end{aligned}
$$

and where

$\sigma_{\varepsilon p}$ is the electric conductivity for an electron-ion gas as derived by Spitzer;

$q_{i j}$ is the cross section for collision of $i$ th and $j$ th particles;

$f_{i i}$ is the correction term to binary diffusion coefficient between particles of type $i$ and $j$ obtained by using the second approximation of the Chapman-Enskog expansion; and

$k_{i}$ is the thermal diffusion ratio of the $i$ th component.

These equations apply over the range of ionization $0<n_{I} / n_{a}<100$. Other relations are necessary to reduce these equations to those for a fully ionized gas.

Combining Eqs. (1) and (2) in the form $|e|\left(n_{I} \mathbf{u}_{I}-n_{e} \mathbf{u}_{e}\right)$ the current density is obtained. On comparing the result with the expressions obtained by other authors, ${ }^{2,4-6}$ a number of comments can be made.

(1) The effects of gradients of ion and atom density as well as deviations from charge neutrality are introduced explicitly for the first time to the author's knowledge. These relations are essential for the understanding of phenomena that occur at all interfaces between plasmas and solid boundaries, and in particular at the electrodes of gas discharges.

(2) The effect of magnetic fields on the flow of current and mass flux of charged particles is shown to be considerably more complicated than previously indicated..$^{4,6}$ In particular, there is a current in the direction of $(\mathbf{E} \times \mathbf{B}) \times \mathbf{B}$ in addition to the direct and Hall currents. It is shown that the equations derived can be put into a form similar to the equations of Chapman and Cowling ${ }^{4}$ for the interaction of a magnetic field with the flow of electrons and ions, the only difference being linear terms coupling the ion and electron velocities. These coupling terms can be neglected only when the percent ionization is very low $\left(<10^{-2}\right)$ and not in all cases even then. These coupling terms implicitly apply the constraint of zero mass motion of the gas locally to the equations describing the electron and ion motion as derived by Chapman and Cowling, and hence remove a discrepancy pointed out by them concerning their equations, which were derived from mean free path considerations.

In order to see more clearly how Eqs. (1) and (2) differ from similar expressions of other authors, they can be written in a more suitable form. After some manipulation of Eqs. (1) and (2) we obtain the following expressions:

$$
\begin{aligned}
\mathbf{u}_{e}=-\gamma\left[\frac{(\beta+\epsilon) \eta-\epsilon}{\eta}\right]\left(\mathbf{F}_{1}+\mathbf{u}_{e} \times \mathbf{B}\right)+\frac{\mathbf{u}_{I}}{\eta} \\
\mathbf{u}_{I}=\gamma\left[\frac{(\beta+\epsilon) \eta-\epsilon}{\beta+\epsilon}\right] \\
\cdot\left(\mathbf{F}_{1}-\mathbf{F}_{2}+\mathbf{u}_{I} \times \mathbf{B}\right)+\frac{\epsilon \mathbf{u}_{e}}{\beta+\epsilon} .
\end{aligned}
$$

Except for the last term on the right-hand side in each of Eqs. (3) and (4), these expressions can be identified with Eqs. (6) and (7), page 328 of Chapman and Cowling. ${ }^{4}$ The conductivity tensors of Allis and Buchsbaum 6 express essentially the same relationships as are found in Chapman and Cowling." As was mentioned previously, the addition of the last term on the right-hand side of Eqs. (3) and (4) results from the requirement that $\mathbf{u}_{0}$ and $\mathbf{u}_{I}$ be diffusion velocities with respect to the local mass velocity of the gas.

In using Eqs. (1) and (2) and applying boundary conditions valid at plasma-solid interfaces, it has been found possible to explain a number of puzzling phenomena that occur at arc electrodes without having to postulate any new mechanism. In particular, the following phenomena have been treated: (1) contraction of arc cathode and anode spots; (2) transmission from field emission to thermionic emission at the cathode; (3) retrograde motion of the cathode spot in a magnetic field.

Details of these investigations, together with an analytic and experimental investigation of the energy transfer processes from electric arcs can be found in reference 1. Technical papers are being prepared on some aspects of this work.

This work was carried out under the sponsorship and with the financial assistance of the Office, Chief of Ordnance, and the Office of Ordnance Research. U. S. Army.

${ }^{1} \mathrm{G} . \mathrm{L}$. Cann, "Energy transfer processes in a partially ionized gas." Ph. D. thesis, California Institute of Technology, Pasadena, California, 1960.

${ }^{2}$ Handbuch der Physik, edited by S. Flügge (SpringerVerlag, Berlin, 1956), Vol. 22, pp. 254-444.

${ }^{3}$ J. O. Hirschfelder, C. F. Curtiss and R. B. Bird, Molecular Theory of Gases and Liquids (John Wiley \& Sons, Inc. New York, 1954), pp. 712-716.

${ }^{4}$ S. Chapman, and T. G. Cowling, The Mathematical Theory of Non-Uniform Gases (Cambridge University Press, New York, 1952).

B L. Spitzer, Physics of Fully Ionized Gases (Interscience Publishers, Inc., New York, 1956), pp. 21, 84.

${ }^{6}$ A. B. Cambel, and J. B. Fenn. Dynamics of Conducting Gases (Northwestern University Press, Evanston, Illinois, 1960), pp. 3-14.

\section{Effect of Many-Body Collisions on the Rate of Thermonuclear Reactions}

\author{
Howard S. TaYLOR \\ Universite Libre de Bruxelles, Brussels, Belgium
}

(Received July 5, 1960)

T HAS been shown that the rate of reaction of highenergy particles in a plasma forms the main contribution to the total thermonuclear reaction rate. ${ }^{1}$

Since collective phenomena also are associated with high-velocity particles, a question as to the relation between the two phenomena arose. By using Grad's ${ }^{2}$ method of estimation of the perturbation in the tail of the Maxwellian ion distribution function in a plasma, the 